\title{
Accelerated rates of forgetting of spatial information during aging and long-term ethanol consumption in mice: Evidence for two distinct forms of amnesia
}

\author{
DANIEL J. BERACOCHEA, ANTOINE N. TAKO, and ROBERT JAFFARD \\ Université de Bordeaux I, Talence, France
}

\begin{abstract}
In the experiments reported here, we examined the effects of aging and long-term alcohol consumption on spatial memory performance of $\mathrm{BALB} / \mathrm{c}$ mice in a $\mathrm{T}$-maze. The spontaneous alternation task used consisted of two forced trials (acquisition phase) followed, at varying retention intervals (from $5 \mathrm{~min}$ to $6 \mathrm{~h}$ ), by a free-test trial (retention phase). Tests were conducted under different conditions by varying the intramaze contexts during the retention phase only. Thus, relative to acquisition trials, animals were submitted to test trials either involving a change of intramaze context (cue added: $\mathrm{ON}$ ) or being run in their usual condition (no context variation: OFF). The results of the first experiment showed that in usual context conditions (OFF), both aging (17-month-old subjects) and an administration of alcohol over a 6-month period (9-monthold subjects) induced an accelerated rate of decay of spontaneous alternation as a function of delay. The results of the second experiment showed that a change in context (ON; 6-h retention interval) dramatically alleviated the deficit observed among alcohol-treated subjects, whereas aged animals were still impaired. The results of the first experiment indicate that both aged and alcohol-treated mice exhibited an accelerated rate of "forgetting" as defined operationally. However, the results of the second experiment suggest that the deficit of alcohol-treated animals under usual conditions (OFF) is probably attributable to an impairment of retrieval processes, since the deficit was reversed by the context change. In contrast, the persistence of the deficit among aged mice suggests that their deficit was more likely related to an impairment in the maintenance of information over time.
\end{abstract}

There exist clear parallels between certain cognitive changes associated with alcohol abuse and those associated with aging. These similarities have led to the speculation that prolonged alcohol abuse results in "premature aging" of the brain (Ryan, 1982). However, more detailed neuropsychological investigations have shown that there is only partial overlap between the effects of alcohol and aging (Becker, Butters, Hermann, \& D’Angelo, 1983). Structural brain damage and associated memory deficits have also been extensively studied in both alcohol-treated animals (see Walker, Hunter, \& Abraham, 1981) and aged subjects (see Bartus \& Dean, 1981). To our knowledge, however, no direct behavioral comparison between these two groups has yet been investigated.

In previous experiments, we demonstrated that longterm ethanol consumption induced an accelerated rate of decay of spontaneous alternation (SA) in a T-maze as a

The research presented here was supported by a grant from the Haut Comite d'Eude et d'Information sur l'Alcoolisme (1984-36) and by the Centre National de la Recherche Scientifique (URA 339). We thank Thomas Durkin and Frances Ash for their help in writing this manuscript. Correspondence may be addressed to D. J. Beracochea, Laboratoire de Psychophysiologie, UA CNRS 339, Université de Bordeaux I, Avenue des Facultés, 33405 Talence, Cedex France. function of the delay (from $30 \mathrm{sec}$ to $24 \mathrm{~h}$ ) between two forced trials used as an acquisition phase and a free trial used as a retention test (Beracochea \& Jaffard, 1985). We suggested that this phenomenon reflected an accelerated rate of forgetting of spatial information. The results from subsequent behavioral analyses using a paradigm involving a large interference component (i.e., a sequential alternation procedure) led us to speculate that the alcoholinduced deficit may be accounted for by a failure to retain the order of events in memory, that is, a memory deficit for temporal context (see Beracochea, Lescaudron, Tako, Verna, \& Jaffard, 1987). Consequently, a novel contextual cue attached to a given test would considerably improve performance on the particular test (see Winocur, Moscowitch, \& Witherspoon, 1987). The results from those experiments have demonstrated that alcohol-treated animals did, in effect, benefit from such a procedure but in quite an unexpected way. Indeed, providing the animals with new contextual cues at the time of retention totally alleviated their deficit, thereby suggesting an impairment of retrieval processes (Beracochea et al., 1987; see Beracochea \& Jaffard, 1987, for similar results among mammillary-body lesioned animals).

The present experiment had two main aims: (1) to investigate whether aging results in an accelerated rate of 
forgetting, in line with our previous observations on the effects of long-term ethanol consumption (Beracochea \& Jaffard, 1985); and (2) to determine whether a context change occurring at the time of testing could differentially-if at all-help alcohol-treated animals and aged subjects, in terms of remembering information given during the acquisition phase.

\section{METHOD}

\section{Subjects}

The subjects were 132 experimentally naive male mice of the BALB/c strain, approximately 6 weeks old at the time of receipt. They were housed in cages of 15 subjects and were placed in a constant-temperature room $\left(23^{\circ} \pm 1^{\circ} \mathrm{C}\right)$ maintained on a $12: 12$ light:dark cycle (7:00 a.m. to 7:00 p.m.). The animals had free access to food and water for 3 weeks before the beginning of the period of alcohol administration. One week after alcohol was withdrawn and 4 weeks before testing began, the animals were placed in individual cages with ad-lib feeding and watering.

\section{Alcohol Administration}

The method used has already been described in full (see Beracochea \& Jaffard, 1985; Beracochea et al., 1987). The animals assigned to the alcohol groups were supplied with a $12 \%(\mathrm{~V}: \mathrm{V})$ solution of ethanol supplemented with saccharose $(3 \mathrm{~g} / \mathrm{l})$ as their sole source of fluid. Dry food was freely available throughout the experiment. Starting at 9 weeks of age, the treatment was administered over periods of 6 weeks, 6 months, 9 months, and 12 months, according to the groups. At the end of these periods, tap water was substituted for ethanol. Two control groups were used. Mice in the first control group were pair-fed with an isocaloric solution of dextirmaltose and dry food. The animals assigned to the second control group had ad-lib access to food and water. Finally, tests were conducted on four groups differing in age $(4,9,12$, and 17 months, respectively). These groups were, in turn, divided into three subgroups depending on the treatment they had received (i.e., alcohol, pair-fed, and tap water).

\section{Behavioral Testing}

Apparatus. All tests were run in a T-maze made of gray Plexiglas. Stem and arms were $35 \mathrm{~cm}$ long, $10 \mathrm{~cm}$ wide, and $20 \mathrm{~cm}$ high. The startbox $(10 \times 12 \mathrm{~cm})$ was separated from the stem by a vertical sliding door. Horizontal sliding doors were placed at the entrance to each arm.

Procedure. Testing was conducted between 9 a.m. and 5 p.m. Prior to behavioral testing, the subjects were accustomed to being handled on a daily basis (about $5 \mathrm{~min} /$ day) and were exposed to two free-exploration sessions of $5 \mathrm{~min}$ each in the apparatus, in order for them to familiarize themselves with the conditions they were to encounter during the alternation test. Each mouse was then submitted either daily or every $48 \mathrm{~h}$ to a test consisting of two forcedacquisition trials, followed by a free-test trial given either $5 \mathrm{~min}$ or $6 \mathrm{~h}$ later. On acquisition, the mouse was placed in the startbox; after $30 \mathrm{sec}$, the door to the stem was opened and the mouse was forced to enter one of the arms (access to the other being blocked by the sliding door). After a 30 -sec confinement period in the arm, the mouse was removed and placed in the startbox for a second identical trial, during which the mouse was forced to enter the arm visited during the first forced trial. The same procedure was observed on the test trial, except that the mouse had free access to both arms (free trial). Following completion of the free trial, the mouse was allowed to explore the maze for $5 \mathrm{~min}$, during which time the subject could freely enter both arms. This procedure was used to reinforce the familiarity of the normal intramaze context. Regardless of the conditions encountered in the immediately preced- ing test (cardboard or no-cardboard, see below), this exploration session was conducted without the cardboard (for a more detailed description, as well as additional explanations, see Tako, Beracochea, \& Jaffard, 1988). Finally, each animal was tested four times at each retention interval according to the following sequence: $5 \mathrm{~min}$ (twice), $6 \mathrm{~h}$ (four times), and $5 \mathrm{~min}$ (twice). Moreover, the direction in which a given subject was forced to go during the acquisition phase alternated on consecutive tests so that turn biases were eliminated (chance level: 50\%). Twelve- and 17-month-old animals were submitted to four additional tests at the 6-h interval. In these tests, the intramaze context was modified on the test trial by placing a white cardboard $(12 \times 19 \mathrm{~cm})$ between the two arms against the wall, perpendicular to the stem (ON condition). These tests alternated with the four tests given at the 6-h retention interval under normal conditions (OFF conditions).

Data analysis. Mixed-design and separate analyses of variance (ANOVAs) were performed with retention interval and context change/no change as the within-groups factors, and age and alcohol consumption as the between-groups factors.

\section{RESULTS}

\section{Effects of Alcohol Treatment and Age under Standard Maze Context Conditions}

Since no differences were observed between the pairfed and water groups (ANOVAs, $F<.5$ ), the data from these two groups were pooled for statistical analysis (control group).

An ANOVA performed over the four levels of age with or without alcohol consumption showed no differences in performance registered at the shortest retention interval (5 min; effects of age, alcohol, and age $\times$ alcohol interaction, $F<0.32$ ). This finding contrasts with the significant effects of these factors on performances at the 6-h retention interval, which significantly decreased as a function of age in both control $[F(3,63)=3.56, p=$ $.0018]$ and alcohol-treated $[F(3,61)=5.53, p=.002]$ groups. However, for these four levels of age, the interaction between aging and alcohol treatment did not reach statistical significance $[F(3,124)=2.13, p=.098]$ even though, globally, alcohol-treated mice exhibited significantly impaired performance relative to their four agematched control groups [alcohol effect at $6 \mathrm{~h}, F(1,124)$ $=15.0, p<.001$ ] and, more importantly, alcohol-treated mice exhibited significantly greater decline than did controls in alternation rates between the 5-min and 6-h intervals $[F(1,124)=10.7, p<.001]$ (Figure 1A). An ANOVA performed on the control animals showed that aging alone also produced a decline in alternation rates between the 5-min and 6-h intervals [age $\times$ retention interval interaction, $F(3,63)=2.94, p=.04$ ]. However, no significant differences were observed between the 4-, 9-, and 12-month-old groups [age $\times$ retention interval interaction, $F(2,45)=0.47$ ]. Only the 17 -month-old group exhibited a significantly greater decline of SA rates between the 5-min and 6-h intervals than did the two younger groups (4 and 9 months, $p<.019$ in both cases). The same nonsignificant tendency was observed when the comparison was made against the 12-month-old group $[F(1,29)=2.59, p=.11]$. Although no age-related differences were observed for the shortest (5-min) inter- 

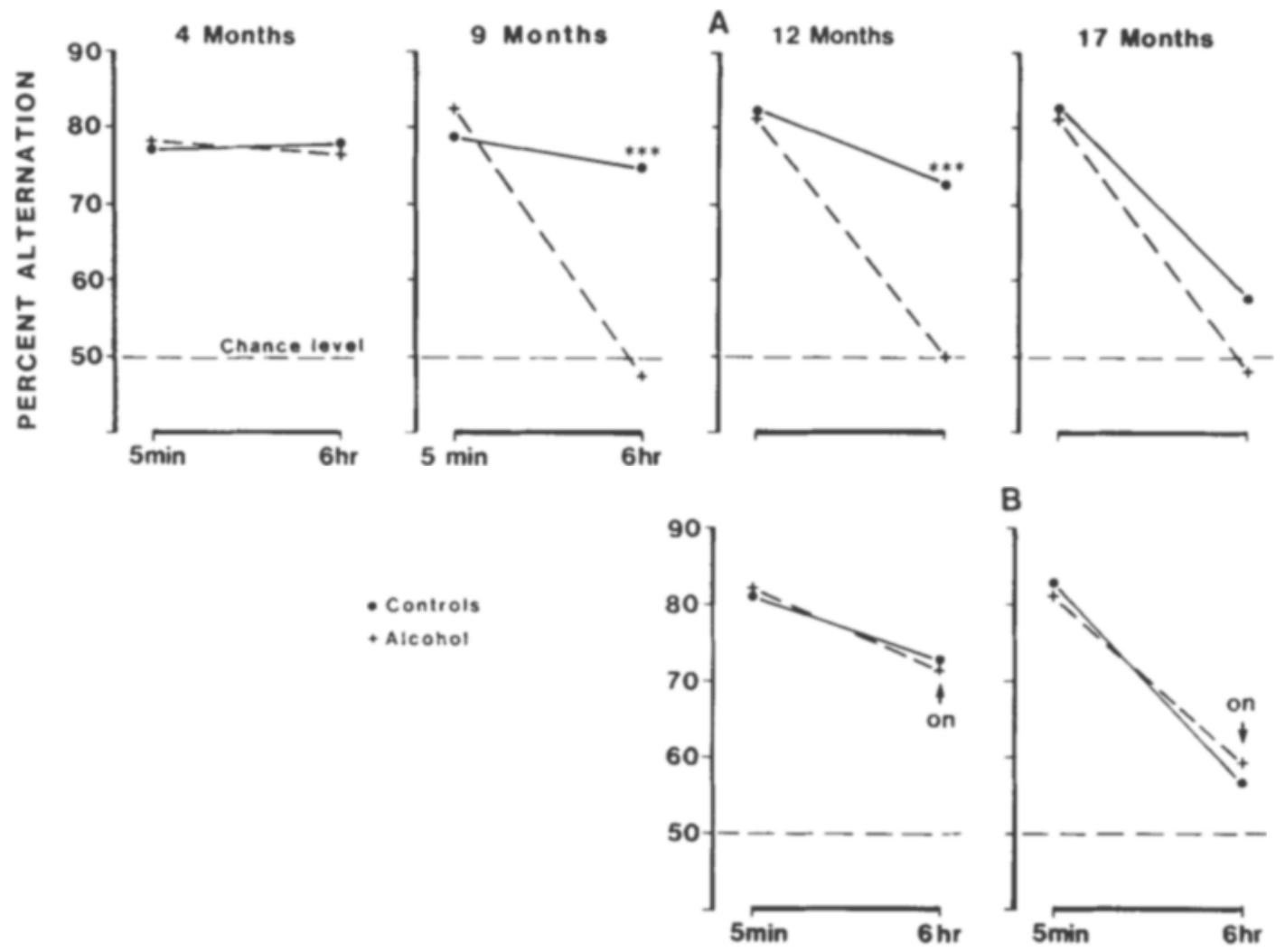

RETENTION INTERVAL

Figure 1. A. Mean percentage of spontaneous alternation under normal conditions (OFF) as a function of retention interval ( 5 min and $6 \mathrm{~h}$ ) at different ages $(4,9,12$, and 17 months) in groups that had (alcohol groups: $n=15,20$, 14 , and 16, respectively) or had not (control groups: $n=16,20,12$, and 19, respectively) received chronic ethanol administration $(1,6,9$, and 12 months). $* * *$ indicates significantly different from the alcohol groups $(p<.001)$. B. Same as above for the 12- and 17-month-old groups, except for the 6-h interval, in which a cardboard was added (ON) on the retention test. The 5 -min sessions were performed under usual (OFF) context conditions (see text for other explanations and statistical analysis).

val (between $78.1 \%$ and $82.9 \%$ ), the 17 -month-old subjects were significantly impaired at $6 \mathrm{~h}$, relative to each of the three younger groups $(p<.02$, for each comparison). A quite similar conclusion can be drawn from the effects of alcohol, with the noticeable difference that the accelerated rate of decay of SA between the 5-min and 6-h intervals was observed earlier. Thus, relative to their age-matched control group, 9-month-old alcohol-treated

Table 1

Percentage of Spontaneous Alternation (mean \pm SEM) in Alcohol-Treated $(n=12)$ and Control $(n=14)$ Mice at 12 Months of Age as a Function of Retention Interval ( 5 min and 24 h) in Normal Conditions (OFF) and, for the 24h Interval, with Varying Intramaze Cues (cardboard added: $O N$ )

\begin{tabular}{cccc}
\hline & & \multicolumn{2}{c}{ Retention Interval } \\
\cline { 3 - 4 } Condition & Group & 5-min & 24-h \\
\hline OFF & Control & $83.3 \pm 4.6$ & $70.8 \pm 6.8$ \\
& Alcohol & $82.7 \pm 4.5$ & $45.8 \pm 6.0$ \\
\multirow{2}{*}{ ON } & Control & & $68.8 \pm 5.4$ \\
& Alcohol & & $66.7 \pm 4.7$ \\
\hline
\end{tabular}

mice exhibited a higher decline of SA rates between the two retention intervals [treatment $\times$ retention interval interaction, $F(1,38)=10.0, p=.002]$, which again resulted from significantly impaired performance at $6 \mathrm{~h}$ $[F(1,38)=10.4, p=.002]$ associated with normal performance at $5 \mathrm{~min}$ (alcohol, $81.3 \%$; controls, $78.8 \%$ ). Thus, between 4 and 9 months of age, alcohol consumption resulted in a significant accelerated decrease of SA rates between the two retention intervals [age $\times$ treatment $\times$ retention interval interaction, $F(1,67)=3.89$, $p=.049$ ]. Finally, alcohol consumption over a 6-month period resulted, at 9 months of age, in the same deficit as that which occurred later (17 months) as a consequence of normal aging.

\section{Effects of Context Change on Retention-Test Performance}

The results are summarized in Figure 1B and Table 1. A comparison of performances observed with and without (OFF) the cardboard added on the 6-h retention test trial among 12-month-old subjects indicated that, in the former condition, performance was significantly improved [con- 
text effect, $F(1,24)=5.34, p=.028]$, but differentially so [context $\times$ group interaction, $F(1,24)=5.58, p=$ $.025]$. Indeed, although no significant effect was observed among control subjects (from $72.9 \%$ to $75 \%$ ), the performance of the alcohol-treated mice was significantly improved [from $50.0 \%$ to $71.4 \% ; F(1,13)=17.3, p<$ $.001]$. As reported in Table 1 , the same procedure used at the 24-h interval led to similar results [context $\times$ group interaction, $F(1,22)=5.39, p=.028$ ]. In contrast, no significant effect of context change (6-h interval) was observed among the 17-month-old groups [context effect, $F(1,33)=0.34$; context $\times$ group interaction, $F(1,33)=$ 1.06]. In other words, the alcohol-induced, but not the age-related, deficit was reversed by the context change.

\section{DISCUSSION}

The present results demonstrate that both long-term alcohol consumption and aging produce an accelerated rate of decay of SA as a function of the interval elapsing between two forced trials used as an acquisition phase and a free trial used as a retention test. One may infer that this decay reflects an accelerated rate of forgetting (as defined operationally), since no difference between groups was observed at the shortest (5-min) retention interval.

The similarity of the deficit observed among adult alcohol-treated mice (i.e., 9 months old) and normal aged animals (i.e., 17 months old) may seem at first sight to reinforce the hypothesis of premature aging (for a review, see Ryan, 1982). Indeed, when tested under usual conditions (OFF), normal mice exhibited a gradual decline with age in their ability to alternate within a 6-h retention interval-a phenomenon similar to that which occurred earlier (9 months vs. 17 months) among mice treated with alcohol over a 6-month period.

However, providing the animals with an unusual intramaze context $(\mathrm{ON})$ at the time of the retention test $(6$ and $24 \mathrm{~h}$ ) totally alleviated the alcohol-induced, but not the age-induced, deficit. This result dramatically refutes the hypothesis of premature aging.

Previous experiments have revealed that both long-term ethanol consumption and mammillary-body (MM) lesions produced spatial alternation deficits in sequential test procedures, as well as over long delays as demonstrated here. Furthermore, in all cases, a context change occurring at the time of retention test was found to alleviate these deficits completely (Beracochea \& Jaffard, 1987; Tako et al., 1988). These findings, along with the results of the present study, suggest that the memory deficits induced by chronic ethanol administration stem from an impairment of retrieval processes which could be linked, at least in part, to the alcohol-induced damage of the MM (Lescaudron, Beracochea, Verna, \& Jaffard, 1984). We recently found that the deficit reported here among alcohol-treated subjects might depend on the effortful requirements of the task. Thus, using MM-lesioned mice as subjects, we found that this deficit was still observed in a rewarded delayed-alternation procedure, but the experimental subjects exhibited no deficits, relative to con- trols, in a win-stay procedure regardless of the delay length (see Beracochea \& Jaffard, 1988). This finding suggests that the deficit of the experimental subjects is mainly observed in "automatic" rather than in "effortful" forms of memory, because the alternation rule requires less "effort" than does a win-stay one (see Di Mattia \& Kesner, 1984). Such a hypothesis is congruent with the presently observed facilitation of memory performance following a contextual variation (ON). Thus, it is conceivable that such a context variation given in test sessions stimulates the effortful processes involved in normal remembering and, particularly as demonstrated here, at the time of retrieval (see Meissner, 1968).

As stated in a previous paper (Tako et al., 1988) the deficits of the alcohol-treated mice are reminiscentalthough not necessarily the consequence-of frontal lobe dysfunction (see Schacter, 1987). One of the principal findings weighing in favor of such a hypothesis is the inability of alcohol-treated (and MM-lesioned animals) to spontaneously alternate in a T-maze when given more than two successive trials, which suggests an abnormal vulnerability to proactive interference (Beracochea et al., 1987; see also Beracochea \& Jaffard, 1985). In contrast, recent experiments have shown that 17-month-old mice did not exhibit any SA deficit (Jaffard, Beracochea, \& Tako, 1987)-a result that confirms previous observations concerning old rats (Zornetzer, Thompson, \& Rogers, 1982). This finding, together with the presently observed lack of effect of the context change on the impairment observed at the 6-h interval, suggests that the memory deficit observed among aged mice differs sharply from that of alcohol-treated animals. Consequently, one may infer that, with respect to the nature of the task we have used, the accelerated rate of forgetting observed among aged animals may indeed reflect a basic failure in the maintenance of the memory trace over a long interval, a failure which, in turn, cannot adequately account for the memory impairment of alcohol-treated mice.

\section{REFERENCES}

Bartus, R. T., DEAN, R. L. (1981). Developing and utilizing animal models in the search for an effective treatment for age-related memory disturbances. In C. G. Gottfries (Ed.), Normal aging, Alzheimer's disease and senile dementia (pp. 231-267). Brussels: University of Brussels Press.

Becker, J., Butters, N., Hermann, A., d D'Angelo, N. (1983). A comparison of the effects of long-term alcohol abuse and aging on the performance of verbal and non-verbal divided attention tasks. $A l$ coholism: Clinical \& Experimental Research, 7, 213-219.

BeracocheA, D. J., \& JAFFARD, R. (1985). Memory deficits subsequent to chronic consumption of alcohol in mice: An analysis based on spontaneous alternation behavior. Behavioral Brain Research, 15, $15-25$.

Beracochea, D. J., JAFFARD, R. (1987). Impairment of spontaneous alternation behavior in sequential test procedures following the mammillary bodies lesions in mice: Evidence for time-dependent interference-related memory deficits. Behavioral Neuroscience, 101, 187-197.

Beracochea, D. J., * JafFard, R. (1988). Spatial memory deficits following the mammillary bodies lesions in mice are dependent of the effortful requirements of the task. Neuroscience Abstracts, 14, 1229. 
Beracochea, D. J., Lescaudron, L., Tako, A. N., Verna, A., \& JAFFARD, R. (1987). Build-up and release from proactive interference during chronic ethanol consumption in mice: $A$ behavioral and neuroanatomical study. Behavioral Brain Research, 25, 63-74.

Di Mattia, B. V., \& Kesner, R. P. (1984). Serial position curves in rats: Automatic versus effortful information processing. Journal of Experimental Psychology: Animal Behavior Processes, 10, 553-557.

JAFFARD, R., BeracocheA, D. J., \& TAKo, A. N. (1987). Accelerated rate of forgetting of spatial information with aging and long-term ethanol consumption in mice: Evidence for two distinct forms of amnesia. Neuroscience Abstracts, 13, 1126.

Lescaudron, L., Beracochea, D. J., Verna, A., \& JafFard, R. (1984). Chronic ethanol consumption induces neuronal loss in mammillary bodies of the mouse: A quantitative analysis. Neuroscience Letters, 50, 151-155.

MEISSNER, W. W. (1968). Learning and memory in the Korsakoff syndrome. Joumal of Neuropsychiatry, 4, 6-20.

RYAN, C. (1982). Alcoholism and premature aging: A neuropsychological perspective. Alcoholism: Clinical \& Experimental Research, 6, 22-30.
SCHACTER, D. L. (1987). Memory, amnesia, and frontal lobe dysfunction. Psychobiology, 15, 21-36.

Tako, A. N., Beracochea, D. J., \& JafFard, R. (1988). Accelerated rate of forgetting of spatial information following mammillary-body lesions in mice: Effects of context change on retention-test performance. Psychobiology, 16, 45-53.

Walker, D. W., Hunter, B. E., \& Abraham, W. C. (1981). Neuroanatomical and functional deficits subsequent to chronic ethanol administration in animals. Alcoholism: Clinical \& Experimental Research, $5,267-281$.

Winocur, G., Moscowitch, M., \& Witherspoon, D. (1987). Contextual cuing and memory performance in brain-damaged amnesics and old people. Brain \& Cognition, 6, 129-141.

ZORNETZER, S. F., THOMPSON, R. F., \& ROGERS, J. (1982). Rapid forgetting in aged rats. Behavioral Neural Biology, 36, 49-60.

(Manuscript received September 29, 1988; revision accepted for publication April 17, 1989.) 